\title{
Ontology Model-based Situation and Socially-Aware Health Care Service in a Smart Home Environment
}

\author{
Haesung Lee and Joonhee Kwon \\ ${ }^{1}$ Department of Computer Science, Kyonggi University \\ San 94-6, Yiui-dong, Yeongtong-ku, Suwon-si, Gyeonggi-do, Korea \\ \{seastar0202, kwonjh\}@kgu.ac.kr
}

\begin{abstract}
With the brilliant advance of ubiquitous technologies, it is possible to provide more smart and pervasive healthcare services in a smart home environment. Despite of these remarkable advances of technologies, there are few smart home-based personalized healthcare services which fully consider not only user's current situation but also user's social relationship. In this paper, we try to model contexts which are generally distributed in smart home environment using the ontology technology. The set of modeled contexts includes home domain-based contexts and social relationship-based contexts. Based on these context models, we can provide fully personalized healthcare service to a specific user in a smart home environment. For verifying the usefulness of the proposed smart home service, we implemented a prototype of healthcare services. Through the demonstration of the developed prototype, we know that using ontology-based context awareness model can provide situation and social-aware health care services in much pervasive way.
\end{abstract}

Keywords: Smart home healthcare service, situation awareness, socially awareness, ontology-based context model

\section{Introduction}

The interests of smart home services in ubiquitous environment are increasing with the advance of ubiquitous technologies such as the sensor network and diverse types of devices. In addition, the need for new personalized health care services that are usable at anytime and anyplace is growing due to these remarkable technologies. Recently, personalized pervasive application has been viewed as an efficient mean to improve human's life. The emerging context-aware computing paradigm offers a unique and real opportunity for us to create pervasive applications and environment specially designed to support the mobile human in ubiquitous environment. Because of the advancement of the sensor technology, it is possible to cognize user's dynamic current context. So, the emerging mobile computing paradigm offers a unique and real opportunity for us to create pervasive applications and to support the user who wants to be provided with cost-effective smart services such as a personalized health care service. Also, in ubiquitous environment, a person could possess or associate with one or more devices such as smart phones, sensors, actuators, or Radio-Frequency Identification (RFID) and tags which have not only various types of functions but also diverse sizes of the display. Beside, these mobile devices have the ability to access the World Wide Web and to communicate with each other through the wireless. In other words, pervasive presence around us of a variety of computing objects are able to interact with each other and cooperate with their neighbors to reach common goals. Ultimately, all the computing devices 
around us could provide each user with personalized health care services considering user's current contexts.

With the advance of ubiquitous computing technologies, people can spend more time in their homes where various types of technologies ideally provides a safe, comfortable environment in which to relax, communicate, learn and be entertains. Increasingly, it is where people connect with friends and family, conduct business, manage resources, learn about the world, and maintain health. Unfortunately, homes today are ill-suited to exploiting the pervasive computing applications. In other words, there are few personalized smart home health care services which fully consider a specific user's situation for providing appropriate recommendation service with each user's current context. Actually, we think existing smart home healthcare services do not fully apply sensor computing. Therefore, we try to provide smart home-based healthcare services to a specific user in pervasive and seamless way using context awareness computing technique.

The rest of the paper is organized as follows. Section 2 describes related works and background related with the proposed method for developing the context awareness-based healthy life assistant platform. Section 3 discusses in detail the structure of the proposed platform. Section 4 presents the implementation of the context awareness-based healthy life assistant platform. Also, in the Section 4, we additionally develop the smart home healthcare system prototype in smart home environment in order to verify the usefulness of our proposed health care platform in the smart home environment.

\section{Related work and background}

\subsection{Personalized Smart Home Services based on Context-aware Computing}

Mark Weiser first introduces the term 'pervasive' which refers to the seamless integration of devices into user's every life [1]. And the term 'context-aware computing' was defined by Schilit and Thimer in 1994 [2]. They described context as 'location of use, the collection of nearby people and objects as well as the changes to those objects over time'. Much of the early work on context-aware systems used similar extensional definitions which defined context by enumerating the constituting parameter. Dey AK et al., enumerate 'location, time of day, season of the year, and temperature'. These definitions are very special and only reflect the types of information that have been used by the researchers in their context-aware applications [3]. Chen and Kotz defined context is the set of environmental states and settings that either determines and an application's behavior or in which an application event occurs and is interesting to the user [4]. Considering personalized content service, context can be considered as any information that can be used to characterize the situation of a specific user.

People spend more time in their homes than in any other space. The home ideally provides a safe, comfortable environment in which to relax, communicate, learn and be entertained. Beside, increasingly, the home is where people connect with friends and family, conduct business, manage resources, learn about the word, and maintain health. With the brilliant advance of various technologies, our homes will be so fully automated and 'smart'. We will spend nearly all our time in the home engaged in leisure activities because digital and robotic agents will have taken over the mundane chores of every life. Many researchers and technologies are more cautious in predicting the future of the home. Hossein et al., propose new approach to help post-stroke patients. To do this , they fuse inertial and Microsoft Kinect sensors to monitor the patient's intake gestures including fine cutting, loading food, and maneuvering the food to the mouth [5]. Bram et al., tackled the problem of establishing connections between devices in a smart home environment by semantic connections. Their prototypes introduce both a tangible and an augmented reality approach towards exploring, 
making and breaking connections. However, these many researches for smart home are bias toward creating automatic or smart home environments that eliminate the need to think about tasks such as controlling heating and lighting, going to the grocery store, scheduling home appliances, and cooking [6-9]. Besides, there are few considerations about the user's social relationships. Therefore, these researches have the limitation of providing fully personalized services to a specific user.

\subsection{Ontology-based Health Care System}

Ontology helps modeling a world phenomenon by first identifying relevant concepts and defining explicitly concepts and related constraints [10]. Ontologies have been experimented in several context-awareness research domains, including e-health. The definition and user of ontology in the medical domain is an active research field, as it has been recognized that ontology-based systems can be used to improve the management of complex health systems [11]. One relevant example is the GALEN ontology which provides reusable terminology resources for clinical systems [12]. Other works have adopted ontologies for managing organizational knowledge and cooperative work among care networks [13, 14]. Also, Bouamarne et al. develop a decision support ontology based on OWL-DL. The ontology is combined within a preoperative risk assessment software system with a DL reasoner in order to provide a number of clinical decision support functionalities, including risk assessment, recommended tests and recommended clinical precaution protocols.

In the domain of context computing, ontologies have been recognized as a key technology for context modeling [15, 16]. Several works have experimented the use of ontologies in context-aware systems. Fook et al. propose a context model which enables the development of sophisticated systems that facilitates caregiving and clinical assessment of dementia patients [17]. Zhang D et. al., present an overall architecture for such a context-aware healthcare system. They considered device self-sensing mechanism, context processing framework and a service operability platform and identified and elaborated [18].

As described above, there are so many researches about ontology-based health care systems. However, despite of increasing interests in smart home-based health care system, there are few researches about the context model for smart home-based health care system. And most existing healthcare systems can not consider social relationships between users and medical experts or other peoples. In this paper, we define context models which are needed for situation and socially-aware health care service in smart home environment.

\section{The Platform for Smart Home Health Care Systems}

\subsection{Architecture}

For providing the personalized healthy life assistant service in smart home environment, it is needed to support various types of pervasive and mobile computing mechanisms which are based on context data. These computing mechanisms provide some methods of adapting to changes in the context, and for collecting context data. So, we propose a platform that 1) handles wide range of contexts which generally occur in the house, 2) collect various types of sensor data and define what situations currently happen at the home space surrounding the specific user, 3) analyzes historical context data and social data to recommend good health assistant services, 4) supports transparent integration of a wide variety of devices to implement interoperability, remote monitoring, and remote programmability.

The architecture of proposed platform, shown in Figure 1, consists of three layers. First, the physical space layer contains the set of available sensors. A physical space is a smart 
home environment that provides context data from its attached entities such as sensors or computing devices.

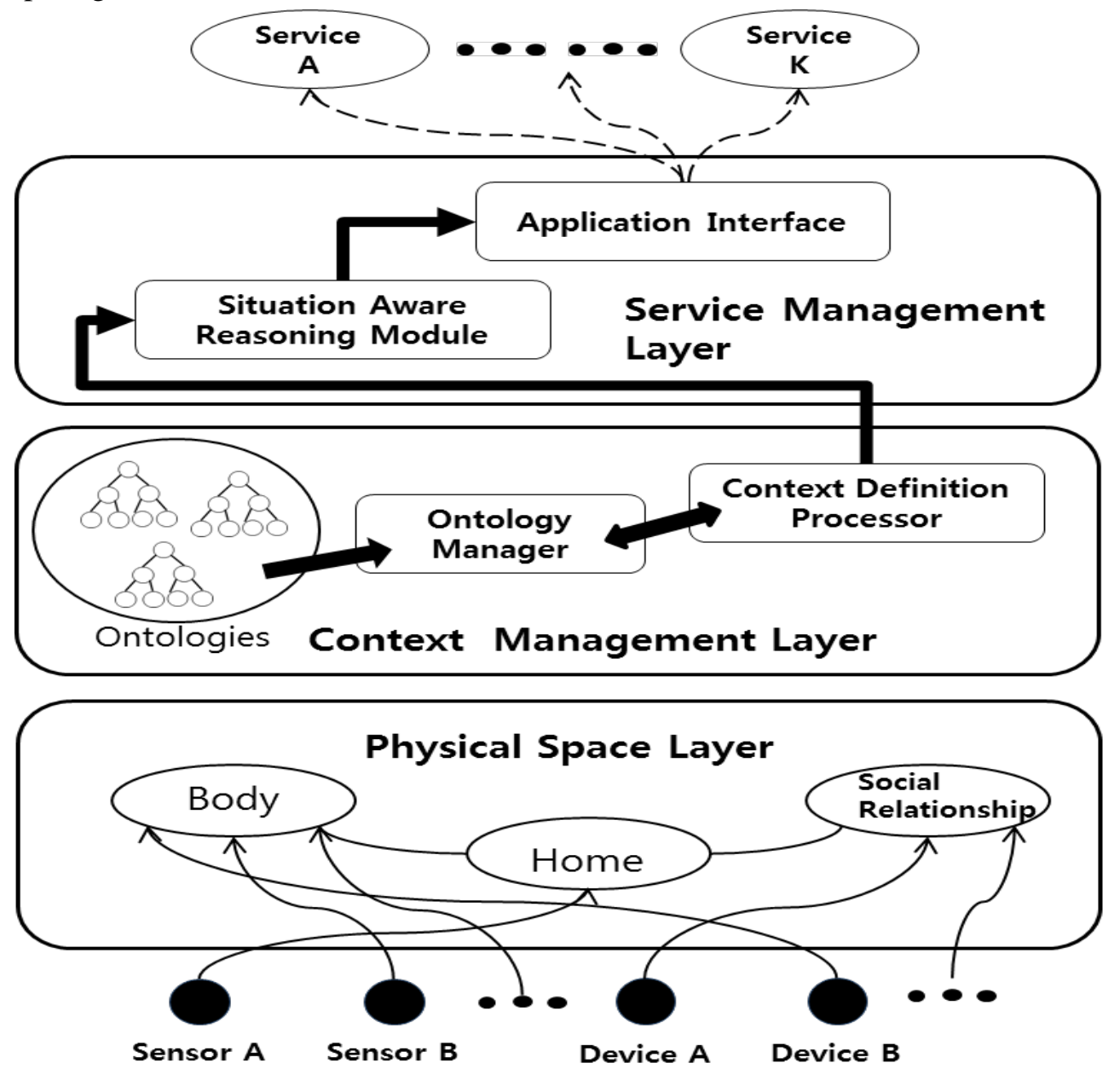

Figure 1. The Platform of Smart Home-based Healthcare System

Second, the context management layer gathers data captured from sensors in the first layer. Sensor data are continuously streamed out of smart home environment into the second layer, "context management layer" which translate law sensor data into contexts which can explain which situation happens around the specific user. After gathering sensor data, context management layer filters sensor data in order to maintain usefully considered data and classify the data into considered context such as location or weather. On top of this layer, context definition processor is implemented for services to acquire considered context data or subscribe to event notifications. Context definition processor interacts with the ontology manager for referring what's mean of the currently captured context. With reference to the methodology for context-ontology modeling proposed in [19], we extended a general-purpose ontology based context model with concepts characterizing basic aspects of a smart-home based healthcare scenario. For example, in the case of health status, the captured context data represent that heart beat is 80 and body heat is $36.5^{\circ} \mathrm{C}$. With these captured contexts, context definition processor inquires about which situations happen to the ontology manager. The ontology manager answers to the question of context definition processor with the fact that 
the human's health status is normal. The ontology manager refers to the knowledge base which is developed with the OWL-based model which describes context-based rules.

Third, the service management layer includes a context-aware reasoning module and an application interface. Context-aware reasoning module is based on the OWL-based model which describes context-based rules that can be used for constructing prioritized service lists. Also, this module provides a way for describing context-triggered actions, e.g., notifications or controlling home appliances. Therefore, different services are triggered through the application interface with the situation aware-based reasoning results.

\subsection{Ontology-based Models}

In the general context model, described in [20], the context of an entity is characterized by one or more context items. We defined three general categories for context items: Biomedical sensor data, Environmental sensor data and Social relationship data. Biomedical sensor data includes heart beats or body heat. Environmental sensor data is consisted of temperature and relative humidity at the user's home. Social relationship data is information regarding members of the smart home-based healthcare network and considered relevant contextual information to be exploited for context-aware assistance service delivery.

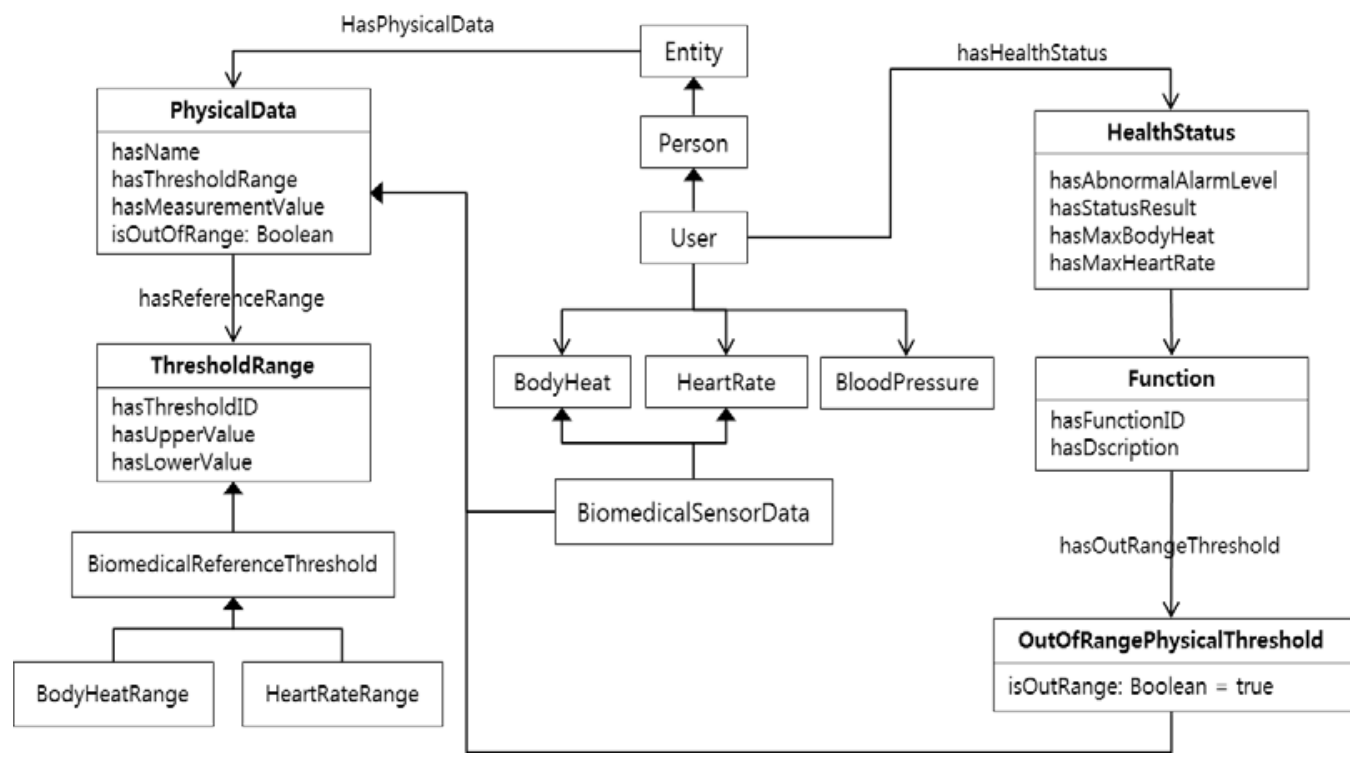

Figure 2. The User Domain Ontology

The specific user's status is derived from context data by applying reasoning rules on measurements of sensor data. In our ontology-based context model include person entities modeling care network members (i.e., user or patient, healthcare experts, family, and emergency technician) and location entities representing symbolic spaces (i.e., living room, kitchen, and bed room). In order to characterize a specific situation, the context model is made of four sub-ontologies: 1) the user domain ontology including entities and relationships for representing monitored physical sensor data for estimating user's health status, 2) the home domain ontology representing monitored environmental parameters, 3) the function management ontology specifying policies for triggering appropriate context-aware services, 
and 4) the social context ontology representing persons involved with different roles in the user's care network.

The user domain ontology includes context data that can be used to define user's health conditions, activity, and position, and to infer possible state notification or abnormal situations. Figure 2 illustrates a diagram of user domain ontology. A specialization of the BiomedicalSensorData is for each considered sensor data (e.g., BodyHeat and HeartRate). Measurement values (hadMeasurementValue) are used to determine user's health status by comparing captured sensor data with a set of predefined reference threshold ranges (ThresholdRange). Each range is specified in terms of upper and lower thresholds. The thresholds are used in a rule-based reasoning when a measured value falls out of the thresholds. We have specified three basic health state alarm levels (very abnormal, abnormal, norm), but the model can be easily extended to include further levels and annotations. The user's HealthStatus is characterized by a hasAbnormalAlarmLevel.

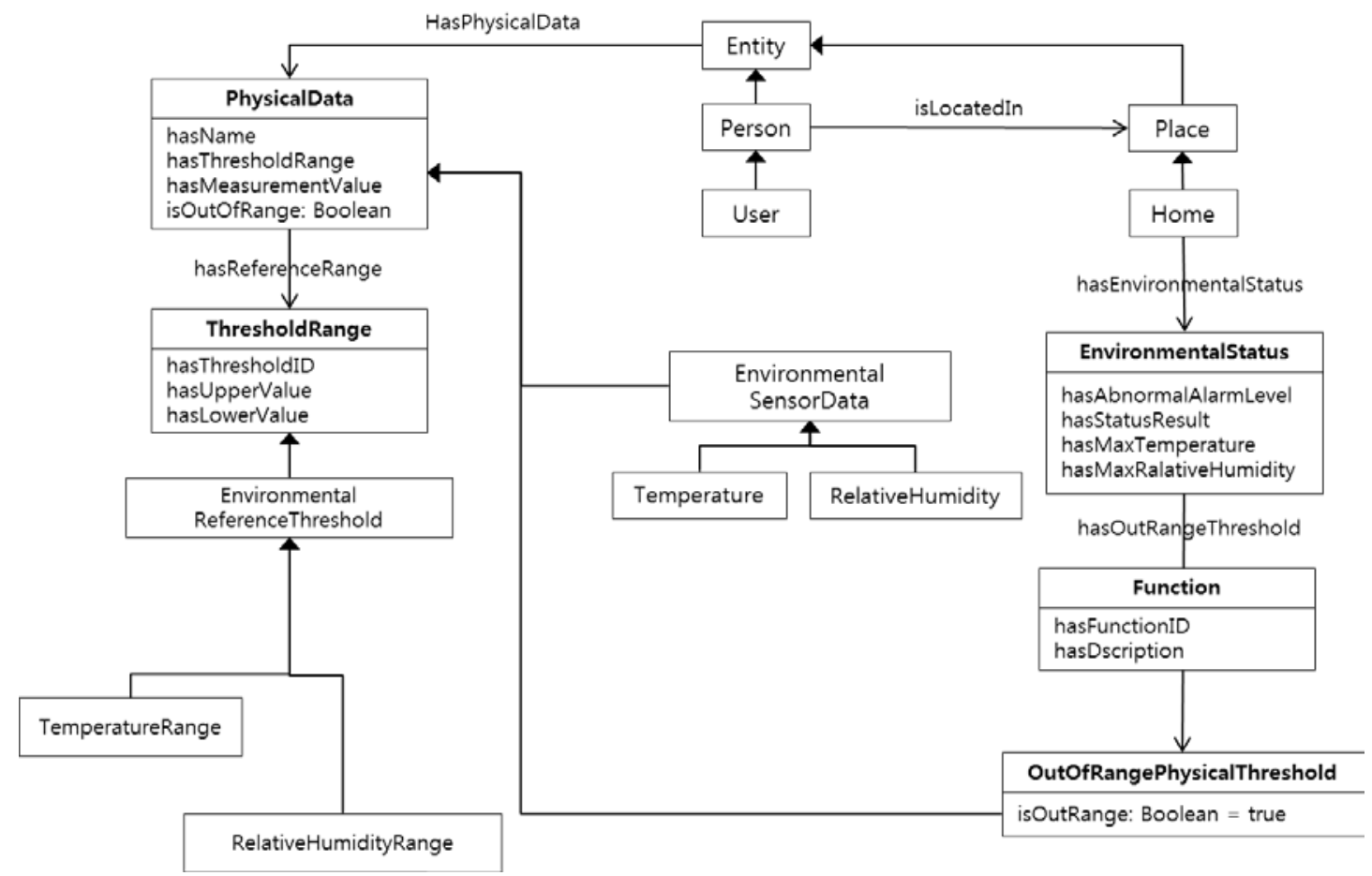

Figure 3. The Smart Home Domain Ontology

In the smart home environment, such as temperature or relative humidity are monitored in order to maintain a healthy environment and detect possible emergency situation (e.g., by means of gas and fire detectors). These situations are modeled by means of the smart home domain ontology, as show in Figure 3. When the system has automatically discerned that a critical situation has occurred, due to abnormal health status or potentially dangerous environmental conditions, proper functions should be done. For example, when the abnormal state representing the too high temperature and the too low humidity is detected, the emergency call service is automatically triggered and the service notifies the emergency status to the nearby fire station. For these situation-based triggering services, we model function policies. More specifically, a function policy specifies when should be triggered. 
In the function management ontology shown in Figure 4, a function policy is modeled as various type of polices (e.g., NotificationPolicy and ControlPolicy). Each policy also specifies related objects \{NotificationPolicy - Person who is notified when the specific situation is happen, ControlPolicy - device, household\}.

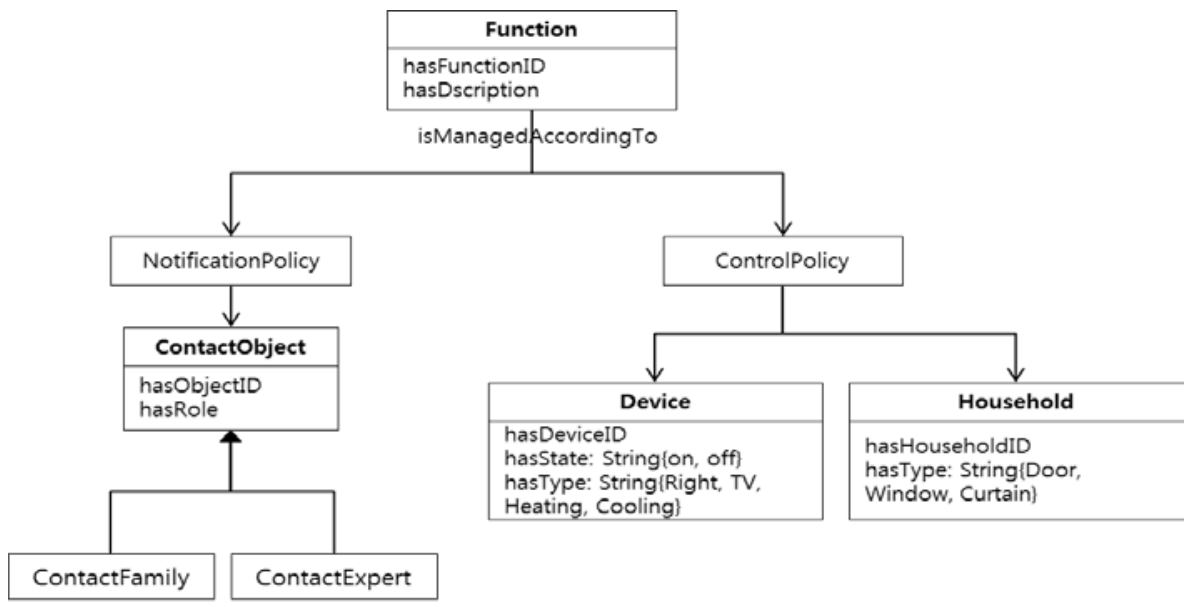

Figure 4. The Function Management Ontology

Members belong to the healthcare network are modeled in the social context ontology shown in Figure 5.

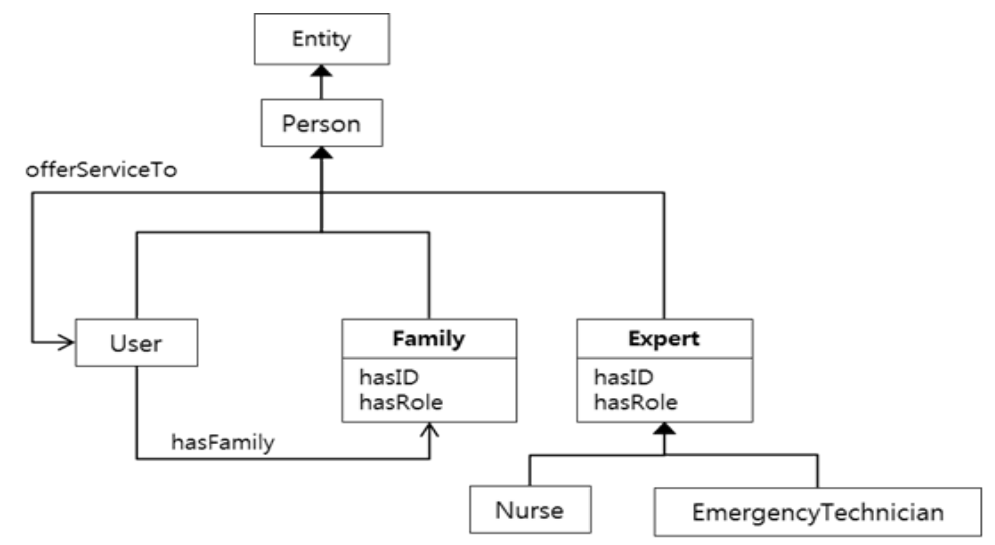

Figure 5. Social Context Ontology

Network members are linked through relationships. In Figure 5, we show only a few relationships, which directly involve the user; a user may have family as a protector (hasFamily), some healthcare expert (offerServiceTo).

\subsection{Reasoning over Ontology Models for Socially and Situation Awareness}

Reasoning over defined context model is triggered for every context data update in order to infer further information on the care network situation (i.e., the user health status, the proper function to be triggered, and the availability of care network members). We define a set of first-order rules as shown in Figure 6(a) in order to determine if a function has to be triggered, 
according to measurement values and thresholds represented in the patient and home domain ontologies.

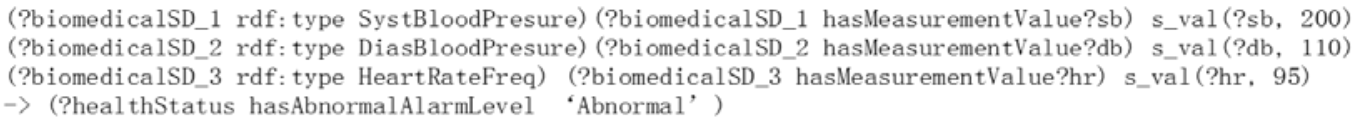

(a) Situation definition

(?function_1 rdf: type Notification) (?function_1 hasFunctionID '12564' ) (?function_1 HasSituation 'Abnormal' ) $\rightarrow$ (?ContactPerson_1 rdf: type Expert) (?person_1 hasID '33124' )

(b) Function definition

\section{Figure 6. The Rule Definition}

For instance, a rule activating a function which notifies an abnormal situation is described as "IF the systolic blood pressure is higher than $200 \mathrm{~mm} / \mathrm{Hg}$, diastolic blood pressure is higher than $110 \mathrm{~mm} / \mathrm{Hg}$ and heart rate frequency is higher than 95 beats/min, THEN the user's health status has an abnormal state level." When the abnormal situation is been detected, the notification function is activated. We defined this function as shown in Figure 6(b).

\section{Prototype Implementation}

This section presents a prototype of smart home-based healthcare system using ontologybased context models. We developed a web-based context generator, smart home server and a mobile client application. Figure 7 shows the overall prototype architecture. The context generator manually generates various contexts which may occur in the home environment. Generated contexts are sent to the reasoning engine which is implemented in the smart home server. Then, through reasoning rules described in the Section 3.3, the reasoning engine triggers a proper function listed in the function module. The function module retrievals function values to the mobile client.

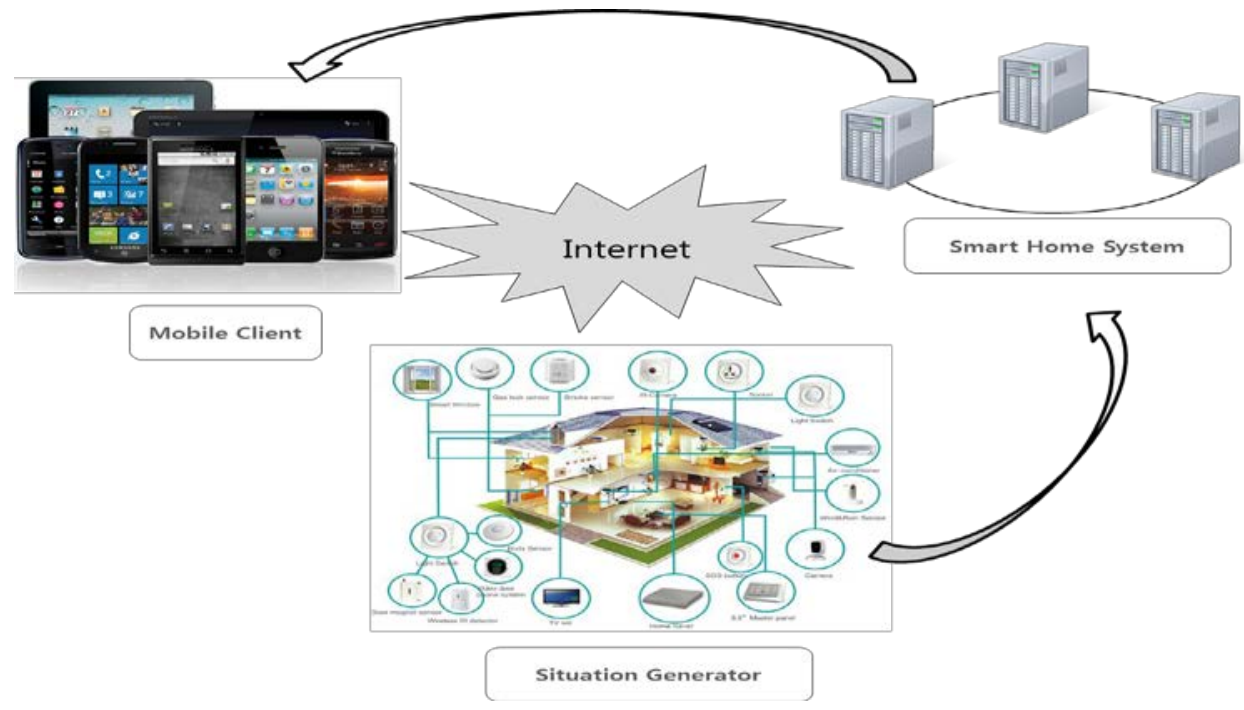

Figure 7. The Prototype Architecture 
Table 1. Implementation Environment

\begin{tabular}{|c|c|}
\hline $\begin{array}{c}\text { Prototype } \\
\text { Component }\end{array}$ & Implementing Tools \\
\hline \multirow{3}{*}{ Smart home server } & $-\quad$ Windows 7 Ultimate, Apache 2.4, JDK 6 \\
& $-\quad$ Protégé-OWL, OWL API for OWL 2.0 \\
& $-\quad$ Intel ${ }^{\circledR}$ Core ${ }^{\mathrm{TM}}$ Quad CPU at 2.4 GHz, and 4 GB \\
Mobile client & $-\quad$ Windows 7 Ultimate, Apache 2.4, JDK 6 \\
\hline Situation generator & $-\quad$ Android SDK 3.0, Samsung Galaxy Play \\
\hline
\end{tabular}

Table 1 describes the implementation environment with which the overall prototype systems are developed. Each prototype component communicates with each other through the internet.

With the implemented context generator, we consider various contexts such as heart rates, body heats, the blood pressure, the temperature of home spaces, home's relative humidity, and user's current location in the house. Based on manually generated contexts, the reasoning engine in the server triggers a proper function. Triggered function controls mobile clients or notified the alarm to proper person such as a family or an emergency technician. Figure 9 shows the user's currently monitored status and mobile client which display the alarm notified from a triggered function. The web-based context generator is for fully building smart home environment. The context generator generates various types of contexts. Figure 8 represents the implemented context generator.

\section{Context generator for Smart home environment}

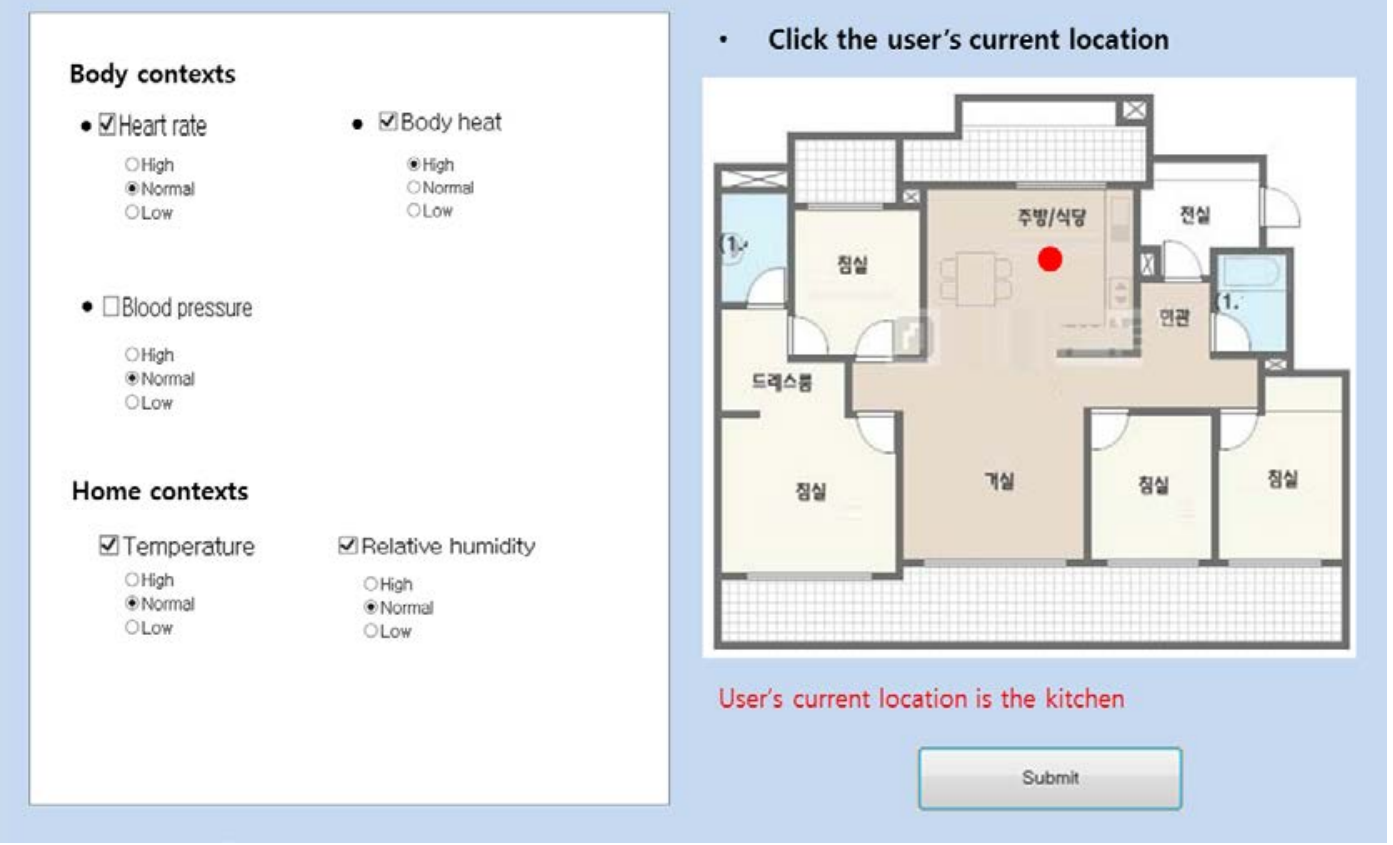

Figure 8. The Context Generator 


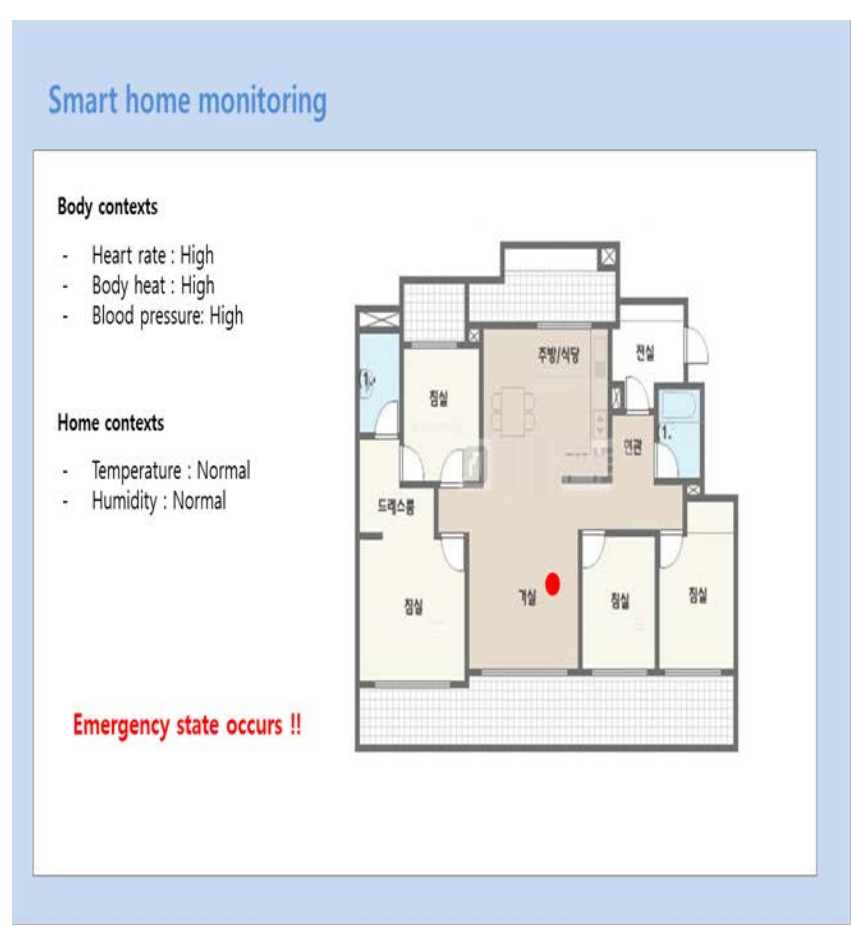

(a)

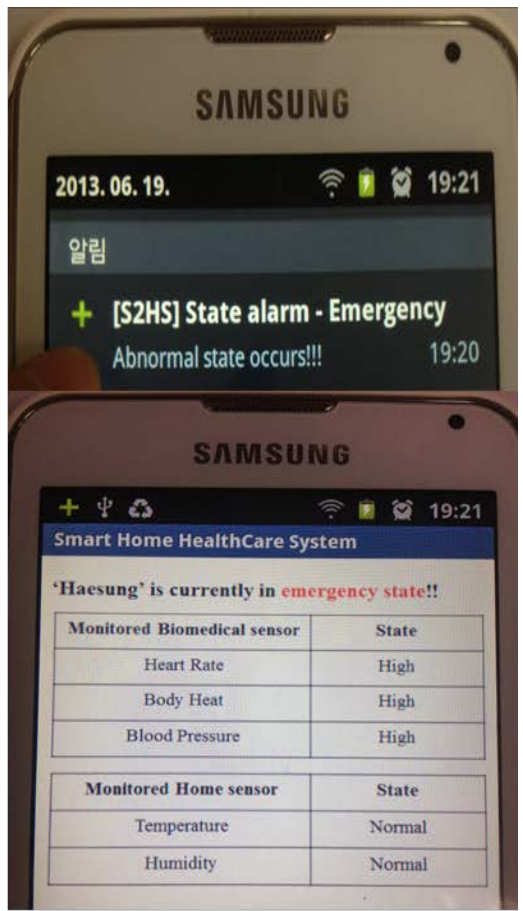

(b)

Figure 9. The Monitored Status (a) and the Emergency Notification (b)

\section{Conclusion}

To date, people can spend more time in their homes where various types of technologies ideally provides a safe, comfortable environment in which to relax, communicate, learn and be entertains. Increasingly, it is where people connect with friends and family, conduct business, manage resources, learn about the world, and maintain health. Unfortunately, homes today are ill-suited to exploiting the pervasive computing applications. In other words, there are few personalized smart home healthcare services which fully consider a specific user's situation and user's social relationships for providing appropriate services with each user's current context.

In this paper, we define the platform for the smart home health care system. Besides, we also define various types of healthcare service ontologies which are carefully considered in smart home environment. For verifying the usefulness of proposed smart home system, we developed smart home system prototype including the context generator and the mobile client.

\section{Acknowledgements}

This work was supported by the Gyonggi Regional Research Center (GRRC) and Contents Convergence Software (CCS) research center.

\section{References}

[1] M. Weiser, “The Computer for the 21st Century”, Scientific American, vol. 265, (1991), pp. 94-104.

[2] Schilit, B., Adams, N. Want, R. Context-Aware Computing Applications. 1st International Workshop on Mobile Computing Systems and Applications, (1994), pp. 85-90. 
[3] A. K. Dey and G. D. Abowd, “Towards a better understanding of context and context-awareness”, CHI'2000 Workshop on the What, Who, Where, When, and How of Context-Awareness, ftp://ftp.cc.gatech.edu/pub/gvu/tr/1999/99-22.pdf, (2000).

[4] G. Chen and D. Kotz, “A survey of context-aware mobile computing research”, Tech. Rep. TR2000-381, Dartmouth, (2000).

[5] H. M. Hondori, M. Khademi and C. V. Lopes, "Monitoring Intake Gestures using Sensor Fusion (Microsoft Kinect and Inertial Sensors) for Smart Home Tele-Rehab Setting”, 2012 1st Annual IEEE Healthcare Innovation Conference, Houston, TX, (2012).

[6] A. Helal, W. Mann, H. El-Zabadani, J. King, Y. Kaddoura and E. Jansen, "The gator tech smart house: A programmable pervasive space”, IEEE Computer, vol. 38, no. 3, (2005), pp. 50-60.

[7] D. J. Cook and S. K. Das, "How smart are our environments? An updated look at the state of the art", Journal of Pervasive and Mobile Computing, vol. 3, no. 2, (2007), pp. 53-73.

[8] M. Darianian and N. Res, Center, Helsinki, Finland, Michael, M. P., Proceeding of 2008 International Conference on Advanced Computer Theory and Engineering, (2008), pp. 116-121.

[9] P.-Y. (Peggy) Chi, J.-H. Chen, H.-H. Chu and J.-L. Lo, "Enabling Calorie-Aware Cooking in a Smart Kitchen”, Persuasive Technology Lecture Notes in Computer Science, vol. 5033, (2008), pp. 116-127.

[10] R. Studer, V. R. Benjamins and D. Fensel, "Knowledge engineering: principles and methods", Data Knowledge. Eng., vol. 25, no. 1-2, (1998), pp. 161-197.

[11] A. Valls, K. Gibert, D. S'ancheza and M. Bateta, "Using ontologies for structuring organizational knowledge in Home Care assistance”, Int. J. Med. Inform., vol. 79, no. 5, (2010), pp. 370-387.

[12] A. L. Rector and J. Rogers, "Ontological and practical issues in using a description logic to represent medical concept systems: Experience from GALEN”, Reasoning Web, Lecture Notes in Computer Science, Springer, (2006), pp. 197-231.

[13] R. Dieng-Kuntz, D. Minier, M. Ruzicka, F. Corby, O. Corby and L. Alamarguy, "Building and using a medical ontology for knowledge management and cooperative work in a health care network", Comput. Biol. Med., vol. 36, no. 7-8, (2006), pp. 871-892.

[14] M. M. Bouamrane, A. Rector and M. Hurrell, "Development of an ontology for a preoperative risk assessment clinical decision support system”, Proc. 22nd IEEE Int. Symp. Computer-Based Med. Syst., (2009), pp. 1-6.

[15] X. H. Wang, D. Q. Zhang, T. Gu, H. K. Pung and H. K., "Ontology based context modeling and reasoning using OWL”, Proc. 2nd IEEE Conf. Pervasive Comput. Commun. Workshops, (2004), pp. 18-22.

[16] T. Strang and C. Linnhoff-Popien, “A context modeling survey”, Proc. Workshop Adv. Context Model., Reason. Manage. (UbiComp 2004), Nottingham, England, (2004).

[17] V. F. S. Fook, S. C. Tay, M. Jayachandran, J. Biswas and D. Zhang, “An ontology-based context model in monitoring and handling agitation behavior for persons with dementia”, Proc. IEEE Conf. Pervasive Comput. Commun. Workshops, (2006), pp. 560-564.

[18] D. Zhang, Z. Yu and C.-Y. Chin, “Context-aware infrastructure for personalized healthcare”, Study Health Technology Information., vol. 117, (2005), pp. 154-163.

[19] X. H. Wang, D. Q. Zhang, T. Gu, H. K. Pung and H. K., "Ontology based context modeling and reasoning using OWL”, Proc. 2nd IEEE Conf. Pervasive Comput. Commun. Workshops, (2004), pp. 18-22.

[20] F. Paganelli, G. Bianchi and D. Giuli, "A contextmodel for context-aware system design towards the ambient intelligence vision: Experiences in the eTourism domain”, Proc. 9th ERCIM Workshop "User Interfaces For All”, Germany, (2006) September 27-28, pp. 173-191.

\section{Authors}

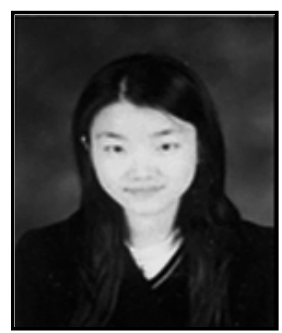

Haesung Lee is a Ph.D. candidate of Computer Science at Kyonggi University, Korea. Her research areas include Context-aware Computing, Social Network, Information Retrieval and Mobile Computing. She works for software development on areas of data search in ubiquitous environment. She received her B.S., M.S. in Computer Science from Kyonggi University,Korea. Contact her at seastar0202@ kyonggi.ac.kr. 


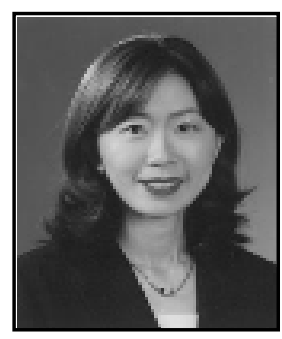

Joon Hee Kwon is an associate professor of Computer Science at Kyonggi University, Korea. She was a visiting research professor at the Computer Science Department at New Jersey Institute of Technology. Her research areas include Context-aware Computing, Information Retrieval, Social Network, Web 2.0 and Mobile Database. Her research projects focus on areas of d1ata search using social network and Web 2.0 in ubiquitous environment. She received her B.S., M.S. and Ph.D. in Computer Science from Sookmyung Women's University, Korea. Contact her at kwonjh@kyonggi.ac.kr. 